\title{
Syndecan-1 induction in lung microenvironment supports the establishment of breast tumor metastases
}

\author{
Colleen Chute ${ }^{1}$, Xinhai Yang ${ }^{1}$, Kristy Meyer ${ }^{1}$, Ning Yang ${ }^{1}$, Keelin O'Neil ${ }^{1}$, Ildiko Kasza ${ }^{6}$, Kevin Eliceiri ${ }^{3,4,5}$, \\ Caroline Alexander ${ }^{3,6}$ and Andreas Friedl ${ }^{1,2,3^{*}}$ (D)
}

\begin{abstract}
Background: Syndecan-1 (Sdc1), a cell surface heparan sulfate proteoglycan normally expressed primarily by epithelia and plasma cells, is aberrantly induced in stromal fibroblasts of breast carcinomas. Stromal fibroblast-derived Sdc1 participates in paracrine growth stimulation of breast carcinoma cells and orchestrates stromal extracellular matrix fiber alignment, thereby creating a migration and invasion-permissive microenvironment. Here, we specifically tested the role of stromal Sdc1 in metastasis.

Methods: The metastatic potential of the aggressive mouse mammary carcinoma cell lines, 4T1 and E0776, was tested in wild-type and genetically Sdc1-deficient host animals. Metastatic lesions were characterized by immunohistochemical analysis.

Results: After orthotopic inoculation, the lung metastatic burden was reduced in Sdc1-/- animals by $97 \%$ and more than 99\%, in BALB/CJ and C57BL/6 animals, respectively. The difference in metastatic efficiency was maintained when the tumor cells were injected into the tail vein, suggesting that host Sdc1 exerts its effect during later stages of the metastatic cascade. Co-localization studies identified Sdc1 expression in stromal fibroblasts within the metastatic microenvironment and in normal airway epithelial cells but not in other cells (endothelial cells, a-smooth muscle actin positive cells, leucocytes, macrophages). The Ki67 proliferation index and the rate of apoptosis of the metastatic tumor cells were diminished in Sdc1-/- vs. Sdc1+/+ animals, and leucocyte density was indistinguishable. Sdc1-mediated metastatic efficiency was abolished when the animals were housed at a thermoneutral ambient temperature of $31^{\circ} \mathrm{C}$, suggesting that the host $\mathrm{Sdc} 1$ effect on metastasis requires mild cold stress.
\end{abstract}

Conclusions: In summary, $\mathrm{Sdc} 1$ is induced in the lung microenvironment after mammary carcinoma cell dissemination and promotes outgrowth of metastases in a temperature-dependent manner.

Keywords: Breast cancer, Proteoglycans, Extracellular matrix, Metastasis, Syndecan, Tumor microenvironment

\section{Background}

The fate of patients with breast carcinoma is determined by distant organ metastasis rather than local disease. Life-threatening metastatic disease is the end-result of a cascade of events that begins with local invasion at the

\footnotetext{
* Correspondence: afriedl@wisc.edu

'Department of Pathology and Laboratory Medicine, University of Wisconsin-Madison, 6051 WIMR, MC-2275, 1111 Highland Avenue, Madison, WI 53705, USA

2Pathology and Laboratory Medicine Service, William S. Middleton Memorial Veterans Hospital, Department of Veterans Affairs Medical Center, Madison, WI, USA

Full list of author information is available at the end of the article
}

primary site and includes intravasation (into blood or lymphatic vessels), survival of tumor cells in the blood stream, extravasation at the distant site and outgrowth of metastatic lesions. Recent experimental evidence suggests that the initial steps of the metastatic cascade including intravasation occur relatively early and that the later events like extravasation and outgrowth may be the rate-limiting steps [1]. Although progress has been made in uncovering the biology of some aspects of metastatic spread, little is known about the mechanisms that govern adaptation of disseminated tumor cells to the environment at the distant site and determine whether tumor

(C) The Author(s). 2018 Open Access This article is distributed under the terms of the Creative Commons Attribution 4.0 International License (http://creativecommons.org/licenses/by/4.0/), which permits unrestricted use, distribution, and 
cells remain dormant or actively proliferate. It is becoming increasingly clear, however, that complex reciprocal interactions between disseminated tumor cells and cells in the local microenvironment (i.e. the metastatic niche) play a crucial role [2].

Syndecan-1 (Sdc1; CD138) belongs to a four-member family of transmembrane heparan sulfate proteoglycans (HSPGs) with roles in cell signaling and adhesion [3]. Sdc1 is primarily expressed by plasma cells and epithelia, including their malignant counterparts. During development, Sdc1 expression is transiently induced in the mesenchyme and the molecule participates in paracrine epithelial-stromal interactions [4]. This mesenchymal induction is recapitulated during malignant progression, when Sdc1 expression is observed in stromal fibroblasts in a variety of carcinoma types, including carcinoma of the breast $[5,6]$. Little is known about the mechanisms of Sdc1 induction in fibroblasts. Induction in mesenchymal cells has been linked to transcriptional regulation by members of the fibroblast growth factor family and extracellular matrix (ECM) constituents [7-9].

Via its heparan sulfate (HS) chains, Sdc1 engages HS-binding ligands including growth factors and many ECM molecules - a property it shares with other HSPGs [10]. Sdc1 core protein-specific binding interactions have been observed between its ectodomain and both integrin cell adhesion receptor subunits and receptor tyrosine kinases [11]. Thus, Sdc1 can act as a cell surface docking station that complexes integrins and receptor tyrosine kinases (RTKs), thereby regulating cell growth and migration. Mice globally deficient in Sdc1 have a surprisingly subtle phenotype with a slight reduction in size and weight noted as the sole abnormality [12]. When the animals are challenged, however, some defects emerge. Sdc1-knockout animals display impaired inflammatory responses, have altered vascular and endothelial cell biology and reduced tumorigenesis [12-15]. Sdc1 deficiency also affects lipid metabolism and reduces tolerance to cold temperatures $[16,17]$. The molecular pathways involved in these impaired responses to external and intrinsic challenges are largely unknown.

In breast cancer, Sdc1 generally acts as a promoter of tumor growth and progression via multiple mechanisms of action. Sdc1 overexpression in human breast carcinoma correlates with a proliferative state and poor prognosis [18-20]. Mice lacking Sdc1 are relatively resistant to Wnt-induced tumorigenesis, demonstrating that Sdc1 is required for efficient tumorigenesis in this model [12]. Sdc1 modulates tumor progression not only by cell autonomous but also by cell non-autonomous mechanisms. The induction of Sdc1 expression in stromal fibroblasts triggers a reciprocal paracrine signaling loop that stimulates mammary tumor growth in vitro and in vivo $[6,21,22]$. Sdc1-expressing stromal fibroblasts also produce an altered ECM that is characterized by parallel, aligned fibronectin and collagen fibers, which is permissive to carcinoma cell migration and invasion and thus has the potential to promote carcinoma spread and metastasis [23]. Collectively, these findings indicate that Sdc1 can stimulate breast tumor progression at many levels.

The goal of the present study was to determine whether host Sdc1 plays a role in mammary carcinoma metastasis. We showed in two mouse strains that the ability of highly aggressive mouse mammary tumor cells to metastasize to the lungs is diminished in mice genetically deficient in Sdc1. The requirement of host Sdc1 for efficient metastasis is observed both after orthotopic (fat pad) and tail vein injection, which suggests that Sdc1 exerts its effect during the later steps of the metastatic cascade; likely during metastatic outgrowth. Elevating the ambient housing temperature to thermo-neutral conditions reduces metastatic efficiency in the wild-type animals to the level seen in the knockout mice suggesting that the Sdc1-dependent mechanism affecting metastasis is regulated by the thermogenic response.

\section{Methods}

Cells, tumor cell inoculations and scoring of metastases

The 4T1 mouse mammary tumor cells were obtained from American Type Culture Collection (ATCC) (CRL-2539) and were cultured in Roswell Park Memorial Institute (RPMI-1640) medium supplemented with $10 \%$ fetal bovine serum (FBS), $2 \mathrm{mM}$ L-glutamine and penicillin/streptomycin at $37^{\circ} \mathrm{C}$ in a humidified atmosphere containing $5 \% \mathrm{CO}_{2}$. E0771 cells were purchased from CH3 Biosystems (Amhurst, NY, USA) and cultured in RPMI-1640 medium supplemented with $10 \mathrm{mmol} / \mathrm{L}$ HEPES and 10\% FBS.

For fat pad injections, mice were anesthetized with isoflurane, and $1 \times 10^{7}$ cells in $10 \mu \mathrm{L}$ serum-free DMEM were injected into the exposed, intact left 4th mammary fat pad as described by Miller [24]. Tumors were allowed to grow for 30 days and then mice were humanely killed. For tail vein injections, mice were anesthetized with isoflurane and $1 \times 10^{5}$ tumor cells in $100 \mu \mathrm{L}$ serum-free DMEM were injected through the tail-vein. After 15 days the mice were humanely killed.

Upon completion of in vivo studies, tissues were fixed for $12-18 \mathrm{~h}$ in $10 \%$ buffered formalin (Fisher Scientific, Waltham, MA, USA) and then processed and paraffin embedded. Hematoxylin and eosin (H\&E)-stained slides were either scanned with an Aperio whole slide scanner (Leica Biosystems) or imaged by stitching individually acquired images in Adobe Photoshop. The image files showing sections of whole lungs were carefully examined and metastatic lesions were circled with an Intuos input device (Wacom) and analyzed using a combination of Photoshop (Adobe) and ImageJ (https://imagej.nih.gov/ij/). The number 
of lesions per mouse, the area of each lesion and the area of total lung tissue were recorded. Tumor burden per mouse was defined as area of lung tissue occupied by metastases divided by total area of lung.

\section{Antibodies, reagents and histological analyses}

The antibodies used for immunolabeling are listed in Table 1. Mouse tissue sections were deparaffinized and for antigen retrieval, sections were boiled with citrate buffer ( $\mathrm{pH}$ 6.0, with $0.05 \%$ Tween 20) for $30 \mathrm{~min}$. In preparation for fibroblast antibody (ER-TR7) labeling, sections of mouse tissues were incubated with proteinase $\mathrm{K}$ working solution $(20 \mu \mathrm{g} / \mathrm{mL}$ in TE buffer, $\mathrm{pH}$ 8.0) for 15 min at $37{ }^{\circ} \mathrm{C}$. After incubation with the primary antibody (overnight at $4{ }^{\circ} \mathrm{C}$ for $\mathrm{CD} 4$ and $\mathrm{CD} 8 ; 1 \mathrm{~h}$ at room temperature for all others) and extensive washes, horseradish peroxidase chromogenic (Ventana) or TSA Plus fluorescence detection kits (Perkin Elmer) were applied following the manufacturers' instructions. Nuclei were counterstained with hematoxylin, 4',6-diamidino-2-phenylindole (DAPI) or Hoechst 33,342 as appropriate. CD4 and CD8 positive $\mathrm{T}$ cells were visualized in mouse lung sections by manual immunolabeling using the ImmPRESS polymer detection system and diaminobenzidine (DAB) substrate.

For the analysis of immunohistochemically labeled slides, at least five images per sample were captured using a brightfield or epifluorescence microscope depending on the label. The signal was quantified using a combination of Photoshop and the National Institutes of Health (NIH) ImageJ software [25]. Lymphocyte densities were determined in the two smallest and the two largest metastases per lung. CD4+ and CD8+ lymphocyte densities were expressed as cells per $1 \times 10^{6}$ pixel (megapixel) on images taken at consistent magnification and resolution.

Tissue sections of breast carcinoma metastases to the lung from seven patients were analyzed by dual-labeling for Sdc1 and the mesenchymal/stromal marker vimentin. After deparaffinization and heat-induced antigen retrieval (EDTA, $\mathrm{pH} 8.5,95-100{ }^{\circ} \mathrm{C}$ for $44 \mathrm{~min}$ ), sections were incubated with anti-Sdc1 antibody $\left(8 \mathrm{~min}, 37^{\circ} \mathrm{C}\right)$. After rinsing, the UltraMap mouse HRP polymer kit (Ventana, Roche, catalog number 760-4313) was applied following the manufacturer's instructions. After denaturing and rinsing, a second round of epitope retrieval under the same conditions was applied and the sections were incubated with prediluted anti-vimentin antibody $\left(16 \mathrm{~min}, 37{ }^{\circ} \mathrm{C}\right)$. After rinsing, HQ mouse polymer (Roche, 760-4814) was applied for $8 \mathrm{~min}$ at $37{ }^{\circ} \mathrm{C}$ followed by anti-HQ HRP solution (Roche, 760-4820; $8 \mathrm{~min}, 37^{\circ} \mathrm{C}$ ) and the Discovery purple detection kit (Roche 760-229).

\section{Second harmonic generation microscopy and collagen fiber analysis}

H\&E-stained histology slides were placed onto an optical workstation built around a Nikon Eclipse TE300 (Nikon, Tokyo, Japan), with a Ti:Sapphire laser (Spectra-PhysicsMillennium/Tsunami, Mountain View CA, USA) excitation source tuned to $890 \mathrm{~nm}$, focused with a $\times 20$ Nikon Plan Apo lens (Nikon, Tokyo, Japan), filtered with a $445 \mathrm{~nm}$ narrow band pass filter (TFI Technologies, Greenfield MA, USA) and back-scattered second harmonic generation (SHG) signal collected with an H7422P-40 detector (Hamamatsu, Japan) and WiscScan acquisition software developed at the Laboratory for

Table 1 Antibodies used for immunolabeling

\begin{tabular}{lllll}
\hline Antigen & Dilution & Specificity & Catalog number & Company/source \\
\hline CD31 & $1: 400$ & Goat anti-mouse & AF3628 & R\&D Systems, Minneapolis, MN, USA \\
Ki67 & $1: 1000$ & Mouse anti-human, clone MIB-1 & M7240 & Dako, Santa Clara, CA, USA \\
Ki67 & $1: 200$ & Rabbit anti-mouse, clone D3B5 & 12,202 & Cell Signaling Technology, Danvers, MA, USA \\
aSMA & $1: 500$ & Rabbit anti-mouse & ab5694 & Abcam, Cambridge, MA, USA \\
F4/80 & $1: 400$ & Rabbit anti-mouse & ab100790 & Abcam \\
ER-TR7 & $1: 200$ & Rat anti-mouse & ab51824 & Abcam \\
CD45 & $1: 200$ & Rabbit anti-mouse & ab10558 & Abcam \\
CD68 & $1: 200$ & Rat anti-mouse & MCA1957T & BioRad \\
Cleaved Caspase-3 & $1: 200$ & Rabbit anti-mouse, D175 & 9661 & Cell Signaling Technology \\
Sdc-1 & $1: 200$ & Rat anti-mouse & NA & gift from Dr. Rapraeger \\
Sdc-1 & $1: 100$ & Mouse anti-human, clone B-B4 & MCA681H & Serotec \\
Vimentin & predilute & Mouse anti-human & $790-2917$ & Ventana Medical Systems, Tucson, AZ, USA \\
CD4 & $1: 200$ & Rabbit anti-mouse & ab221775 & Abcam \\
CD8 & $1: 750$ & Rabbit anti-mouse & ab209775 & Abcam \\
\hline
\end{tabular}


Optical and Computational Instrumentation (LOCI), University of Wisconsin, Madison, WI, USA).

Collagen fiber angles relative to the tumor boundary were analyzed using CTFIRE and the MATLAB-based CurveAlign software developed by the LOCI (http://loci. wisc.edu/software/curvealign) [26]. Intensity of the SHG images was analyzed using the NIH Image) Software (as described above).

\section{Animals}

The generation of Sdc1-/- mice has been described previously [12]. Mice were housed at room temperature $\left(20-23{ }^{\circ} \mathrm{C}\right.$ unless otherwise specified) and maintained on a 12-h light and dark cycle with free access to water and food. For all tumor experiments, 6-8 week old female mice were used. For experiments using thermo-neutral conditions, mice were individually caged and housed at $31{ }^{\circ} \mathrm{C}\left( \pm 1{ }^{\circ} \mathrm{C}\right)$ for 2 weeks in a controlled environment prior to cell inoculation and were monitored daily.

\section{Statistics}

Data are expressed as mean $+/$ - standard error of the mean. Statistical tests were performed using MStat, which is JAVA-based software written at the University of Wisconsin-Madison (http://mcardle.wisc.edu/mstat/) or Prism 7 software (Graphpad). The non-parametric Wilcoxon rank sum, Mann-Whitney or Kruskal-Wallis tests were used unless stated otherwise and $p$ values less than 0.05 were deemed to be significant.

\section{Results}

Host Sdc1 is required for efficient metastasis of mammary carcinoma cells to the lungs

Both carcinoma-cell-associated and stromal Sdc1 can promote breast carcinoma growth $[6,20,22]$ but the importance of this proteoglycan in breast carcinoma progression and metastatic spread is less clear. To determine the role of host Sdc1 in metastasis, we inoculated highly metastatic 4T1 mouse mammary carcinoma cells into the mammary fat pads of syngeneic BALB/cJ wild-type or genetically Sdc1-deficient mice. After 30 days, the number of metastases per mouse (Fig. 1a, b; $p=0.004$ ) and the metastatic burden, defined as percent of lung tissue occupied by metastases (Fig. 1c; $p=0.036$ ) were significantly reduced in Sdc1-/- compared to Sdc1+/+ animals. The average size of metastatic lesions was not significantly different between Sdc1-/- and Sdc1+/+ mice (Fig. 1d; $p=0.11$ ) - presumably because of the small number of metastases in the Sdc1-/- mice and the high variability of the size of metastatic lesions in the Sdc1+/+ mice.

Since tumor behavior can be highly dependent on mouse strain variations [27] and because rate-limiting steps of metastasis may differ between carcinoma cell lines, we tested the effect of host Sdc1 in a different mouse mammary tumor metastasis model. The E0771
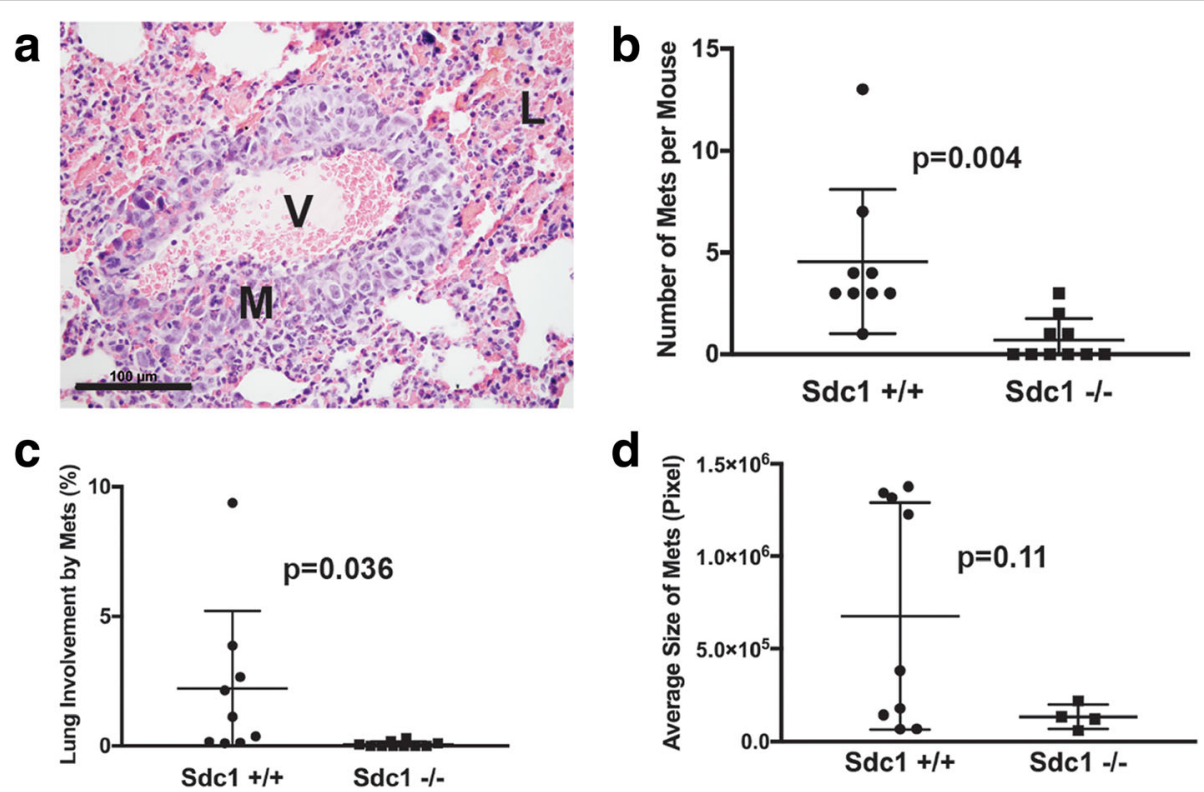

Fig. 1 Effect of host syndecan-1 (Sdc1) on metastatic efficiency of 4T1 mouse mammary carcinoma cells. The $4 \mathrm{~T} 1$ tumor cells $\left(1 \times 10^{7}\right.$ cells in $10 \mu \mathrm{L}$ serum-free DMEM) were injected into the exposed 4th mammary gland as described in "Methods" and mice were sacrificed after 30 days. a Small, early metastasis, cuffing blood vessel in lung ( $M$, metastasis; $V$, vessel; $L$, lung parenchyma; original magnification $\times 400$; scale bar indicates $100 \mu m$ ). b Number of metastatic lesions per mouse. Metastases were counted on single histologic sections of both lungs. Bars indicate mean $+/-$ standard deviation. c Metastatic tumor burden expressed as percent lung tissue occupied by metastatic lesions (see "Methods" for details). d Average area of metastatic lesions expressed in pixels as measured on histologic sections 
mammary tumor cells metastasize primarily to the lungs of $\mathrm{C} 57 \mathrm{BL} / 6$ mice, similar to $4 \mathrm{~T} 1$ cells in BALB/cJ animals [28]. The results with C57BL/6 animals mirrored our observations in BALB/CJ mice, as the number of metastases per mouse (Additional file 1: Figure S1A, B; $p=0.013$ ) and the metastatic burden (Additional file 1: Figure S1C; $p=0.038$ ) were significantly reduced in Sdc1-/- compared to Sdc1+/+ animals. The average size of metastatic lesions was not significantly reduced in Sdc1-/- compared to Sdc1+/+ mice (Additional file 1: Figure S1D; $p=0.37$ ). This result shows that host Sdc1 affects metastatic efficiency independent of mouse strain and cell line and implicates this proteoglycan as a regulator of metastasis.

\section{Host Sdc1 does not significantly affect growth or microenvironmental characteristics of primary tumors}

In the fat pad inoculation model, Sdc1 may affect any step of the metastatic cascade - from local invasion and intravasation, to extravasation and metastatic outgrowth. Previous work by our group and others has shown that Sdc1 is induced in stromal fibroblasts of breast carcinomas in both humans and in mice and that stromal Sdc1 can stimulate tumor growth and angiogenesis and create an invasion-permissive microenvironment [6, 21-23]. However, in this model, the average weight of mammary fat pad tumors grown in Sdc1+/+ or Sdc1-/- mice did not differ significantly (Additional file 2: Figure S2A). The absence of host Sdc1 did not affect the Ki67 proliferation index in the primary carcinoma cells, indicating that host Sdc1 does not significantly stimulate $4 \mathrm{~T} 1$ carcinoma cell proliferation in the primary tumor site (Additional file 2: Figure S2B).

Since stromal Sdc1 has previously been shown to stimulate angiogenesis [22], we studied the tumor vasculature by labeling primary tumor sections with an antibody to the endothelial cell marker CD31. However, in this model, no difference in microvessel density was detected between tumors arising in $\mathrm{Sdc} 1+/+$ or Sdc1-/mice (Additional file 2: Figure S2C). Myofibroblasts or carcinoma-associated fibroblasts (CAF) and macrophages not only stimulate tumor growth but also modulate the metastatic behavior of carcinoma cells $[29,30]$. Therefore, we examined whether host Sdc1 affects these cellular constituents of the local tumor microenvironment. Alpha smooth actin ( $\alpha$ SMA)-positive cells were arranged in a pattern similar to CD31-positive cells, suggesting that most intratumoral $\alpha$ SMA-positive cells are vascular smooth muscle cells or pericytes. The density of intratumoral $\alpha$ SMA-positive cells was similar in Sdc1+/+ and Sdc1-/- host animals (Additional file 2: Figure S2D). We were also unable to detect a difference in the density of intratumoral macrophages (Additional file 2: Figure S2E).
Given the role of stromal Sdc1 in ECM fiber assembly in vitro and in vivo, we measured the amount of collagen in the 4T1 mammary tumors by SHG imaging and collagen alignment by computer-assisted analysis of the SHG images [26, 31]. Collagen fibers were found primarily in the periphery of the tumors aggregating into a "pseudo-capsule" (Additional file 2: Figure S2F). There was no significant difference in the amount of collagen (SHG signal intensity/area; not shown) nor in the mean angles between collagen fibers and the tumor boundary when comparing Sdc1+/+ and Sdc1-/- mice (Additional file 2: Figure S2F). In summary, apart from insignificantly smaller tumor size in Sdc1-/- animals, no Sdc1-related differences were identified in the primary 4T1 mammary fat pad tumors.

\section{Host Sdc1 is important during later stages of mammary carcinoma metastasis}

Since host Sdc1-dependent differences in metastatic efficiency could not be attributed to differences in the primary tumors, we focused our attention to later stages of the metastatic cascade. After tail vein injection of tumor cells - an inoculation method that bypasses the early stages (local invasion, intravasation) of metastatic spread - host Sdc1-dependent differences in metastatic efficiency were maintained (Fig. 2). Specifically, the number of metastases (Fig. 2b), metastatic burden (Fig. 2c) and average size of metastatic lesions (Fig. 2d) were significantly reduced in Sdc1-/- mice compared to Sdc1+/+ animals ( $p<0.0001$ for all comparisons). This observation suggests that host $\mathrm{Sdc} 1$ is important at the distant organ site for any or all of the events occurring late during metastatic spread: extravasation, tumor cell survival, escape from dormancy or outgrowth of disseminated tumor cells; however, it does not exclude the possibility that host Sdc1 in the primary tumor also contributes to metastatic efficiency. Similarly, in the C57BL/6 model, the number of metastatic lesions (Additional file 3: Figure S3A, B; $p=0.045$ ) and the metastatic burden (Additional file 3: Figure S3C; $p=0.037$ ) were lower in Sdc1-/- mice, and the average size of the metastatic lesions was not significantly different (Additional file 3: Figure S3D).

\section{Sdc1 is induced in stromal fibroblasts of mammary carcinoma lung metastasis}

Sdc1 expression is induced in stromal fibroblasts of primary mammary carcinomas in mice and in humans $[5,6]$. In view of our finding that host Sdc1 plays a role in the metastatic niche, we examined whether Sdc1 is induced in stromal fibroblasts in lung metastatic lesions. The stromal compartment is relatively sparse in 4T1-derived lung metastases, yet we observed co-localization between Sdc1 and fibroblasts (identified with antibodies to the fibroblast marker ER-TR7) (Fig. 3a). 

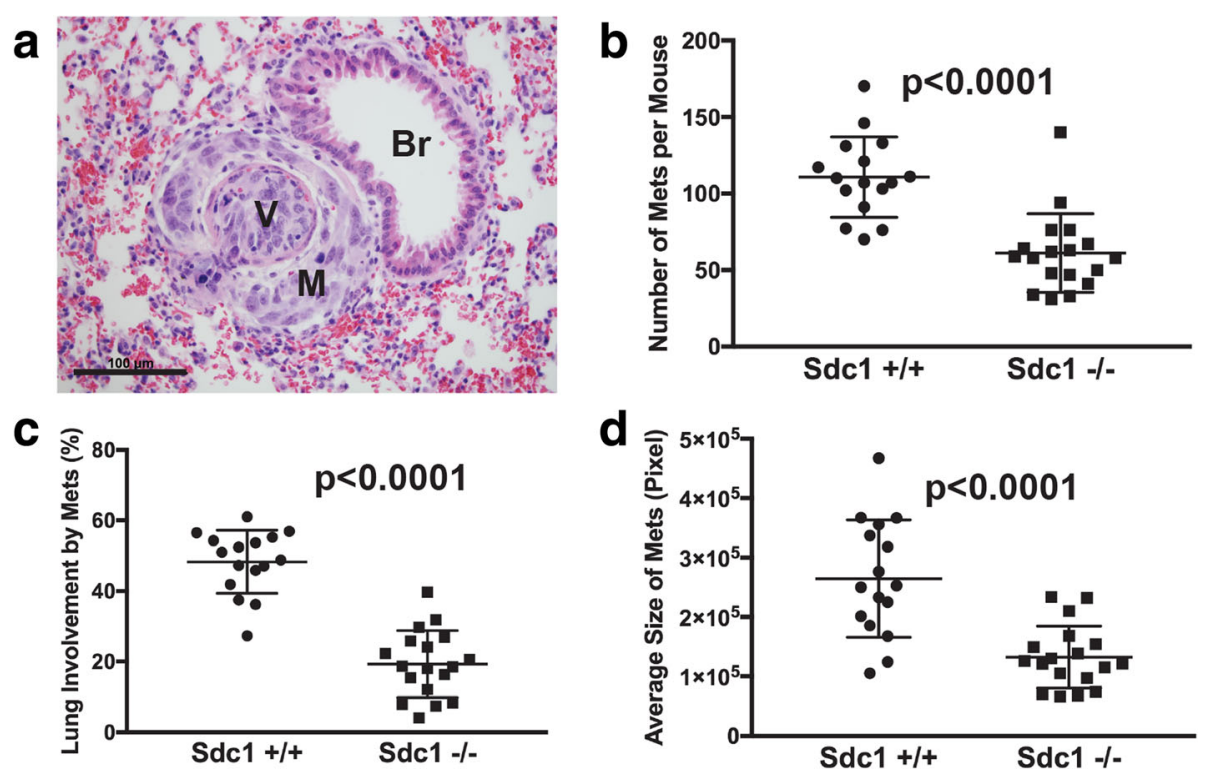

Fig. 2 Effect of host syndecan-1 (Sdc1) on later stages of the metastatic cascade. The $4 \mathrm{TT} 1$ tumor cells $\left(1 \times 10^{5}\right.$ tumor cells in $100 \mu \mathrm{L}$ serum-free DMEM) were injected into the tail vein and mice were sacrificed 15 days later. a Small, early metastasis, with carcinoma cells invading through lung vessel wall and surrounding vessel ( $M$, metastasis; $V$, vessel; Br, bronchiole; original magnification $\times 400$; scale bar indicates $100 \mu \mathrm{m})$. b Number of metastatic lesions per mouse. Metastases were counted on single histologic sections of both lungs. c Metastatic tumor burden expressed as percent lung tissue occupied by metastatic lesions. $\mathbf{d}$ Average area of metastatic lesions expressed in pixels as measured on histologic sections

Sdc1 was absent from (sparse) fibroblasts in normal lung tissue (Fig. 3b). Sdc1 did not co-localize with CD31-positive endothelial cells, nor with $\alpha$ SMA-positive cells, CD45positive leukocytes or CD68-positive macrophages (Fig. 3c-f). Similar to the primary tumors, $\alpha$ SMA-positive cells were found primarily in a vascular pattern, consistent with the distribution of vascular smooth muscle cells or pericytes rather than myofibroblasts. Sdc1 is also present in airway epithelial cells (not shown). Tumor cells express Sdc1 constitutively and as allografts, independent of the mouse host genotype. Biopsy samples from a small collective of patients with breast carcinoma metastases to the lung showed a variable amount of stroma in the metastatic lesions. Stromal fibroblasts in the metastases expressed Sdc1 (Fig. 3g, h), mirroring Sdc1 induction in primary human breast carcinomas. As expected, Sdc1 was also expressed by carcinoma cells in mouse and human samples (Fig. 3c-h).

\section{Loss of host Sdc1 decreases proliferation and apoptosis in metastatic mammary carcinoma cells but does not affect leucocyte density}

As a first step towards determining the mechanism of action of Sdc1 at the lung metastatic site, we further characterized $4 \mathrm{~T} 1$ metastatic lesions in the lungs. Inflammation is considered a driver of tumor progression and different leucocyte populations have been implicated in metastasis. Previous studies have shown that loss of Sdc1 leads to a pro-inflammatory phenotype in the endothelium and enhanced leucocyte recruitment [32, 33]. Also, neutrophils support lung colonization in several mammary tumor models [34] and certain macrophage sub-populations promote mouse mammary tumor metastasis by stimulating extravasation and growth $[35,36]$. In our study, 4T1 lung metastases contained CD45-positive leucocytes primarily in the lesion periphery (Fig. 4a). Leucocyte density was indistinguishable between metastases arising in Sdc1+/+ vs. Sdc1 -/- mice, and therefore it is unlikely that an Sdc1-mediated increase or decrease in inflammation/immune cell infiltration is responsible for differences in metastatic efficiency.

Stromal Sdc1 has been shown to promote breast carcinoma cell proliferation via paracrine pathways $[6,21,22,37]$. Therefore, Sdc1-dependent metastatic efficiency may be due to the stimulation of carcinoma cell proliferation by stromal cell-derived Sdc1. The Ki67 proliferation index in metastatic carcinoma cells was significantly $(p=0.0002)$ decreased by $86 \%$ in Sdc1-/- mice compared to their Sdc1+/+ counterparts (Fig. 4b). The proportion of apoptotic carcinoma cells - identified by active caspase 3 expression - was also lower in Sdc1-/- animals by $64 \%$ (Fig. 4c). Overall, these data are consistent with Sdc1 in the metastatic microenvironment stimulating the proliferation of carcinoma cells. 

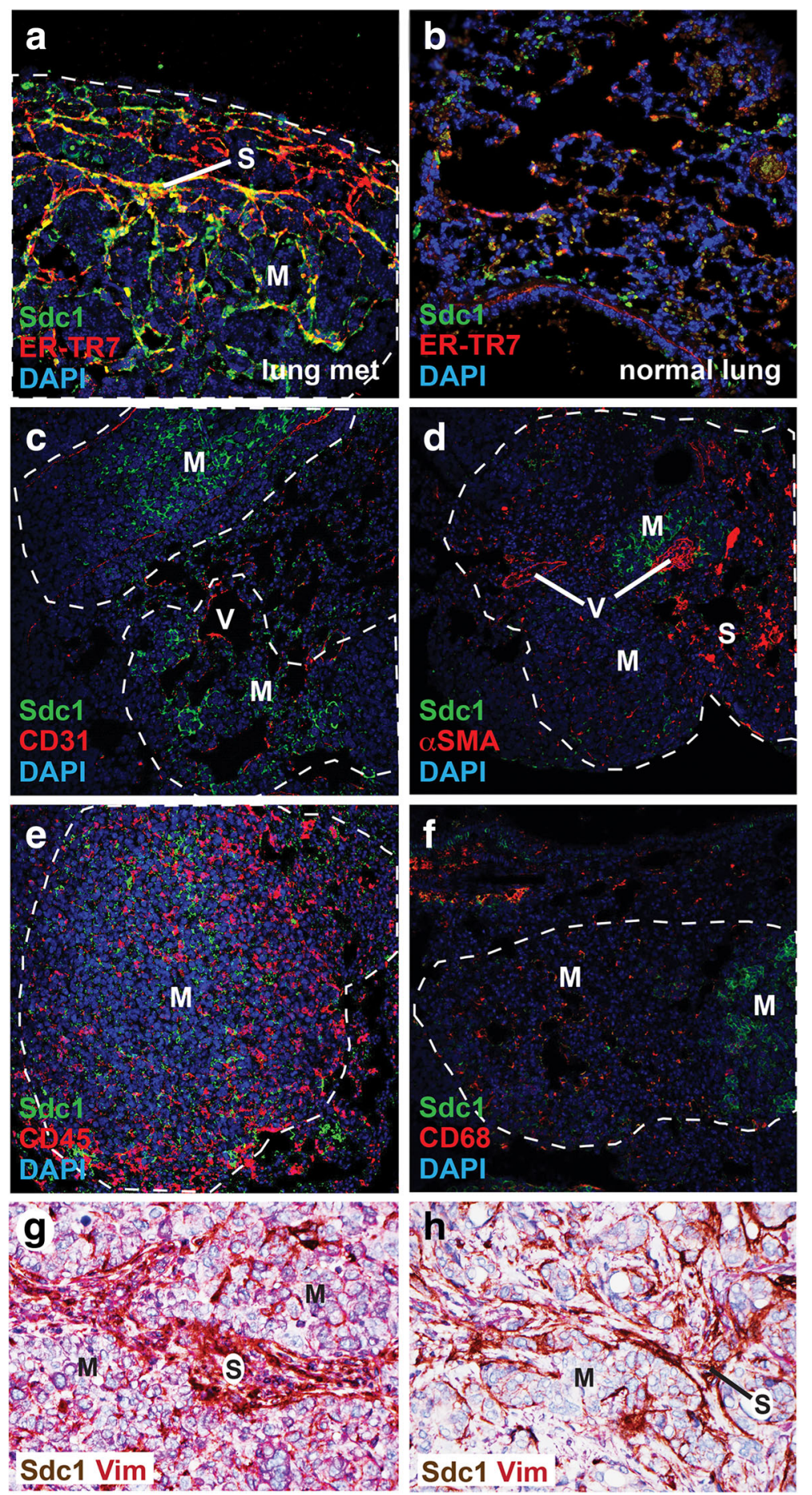

Fig. 3 Syndecan-1 (Sdc1) expression in the metastatic microenvironment. Tissue sections are from tail-vein-injected 4T1 cells in Sdc1+/+ mouse (a-f) or human $(\mathbf{g}, \mathbf{h})$ mammary carcinoma lung metastases unless stated otherwise and were labeled with antibodies to Sdc1 and cell lineage markers as indicated. a Sdc1 (green) and fibroblast marker ER-TR7 (red). b Normal lung tissue labeled for Sdc1 (green) and fibroblast marker ER-TR7 (red). c Sdc1 (green) and endothelial cell marker CD31 (red). d) Sdc1 (green) and myofibroblast/smooth muscle/pericyte marker alpha smooth muscle actin (aSMA) (red). e Sdc1 (green) and leucocyte marker CD45 (red). f Sdc1 (green) and macrophage marker CD68 (red). $\mathbf{g}$ Human breast cancer metastasis to the lung labeled for Sdc1 (brown) and mesenchymal marker vimentin (Vim, magenta). Original magnification approximately $\times 400$ for all images. Metastatic lesions are outlined with dashed lines. M, metastasis; S, stroma; V, vessel; DAPI, 4',6-diamidino-2-phenylindole 

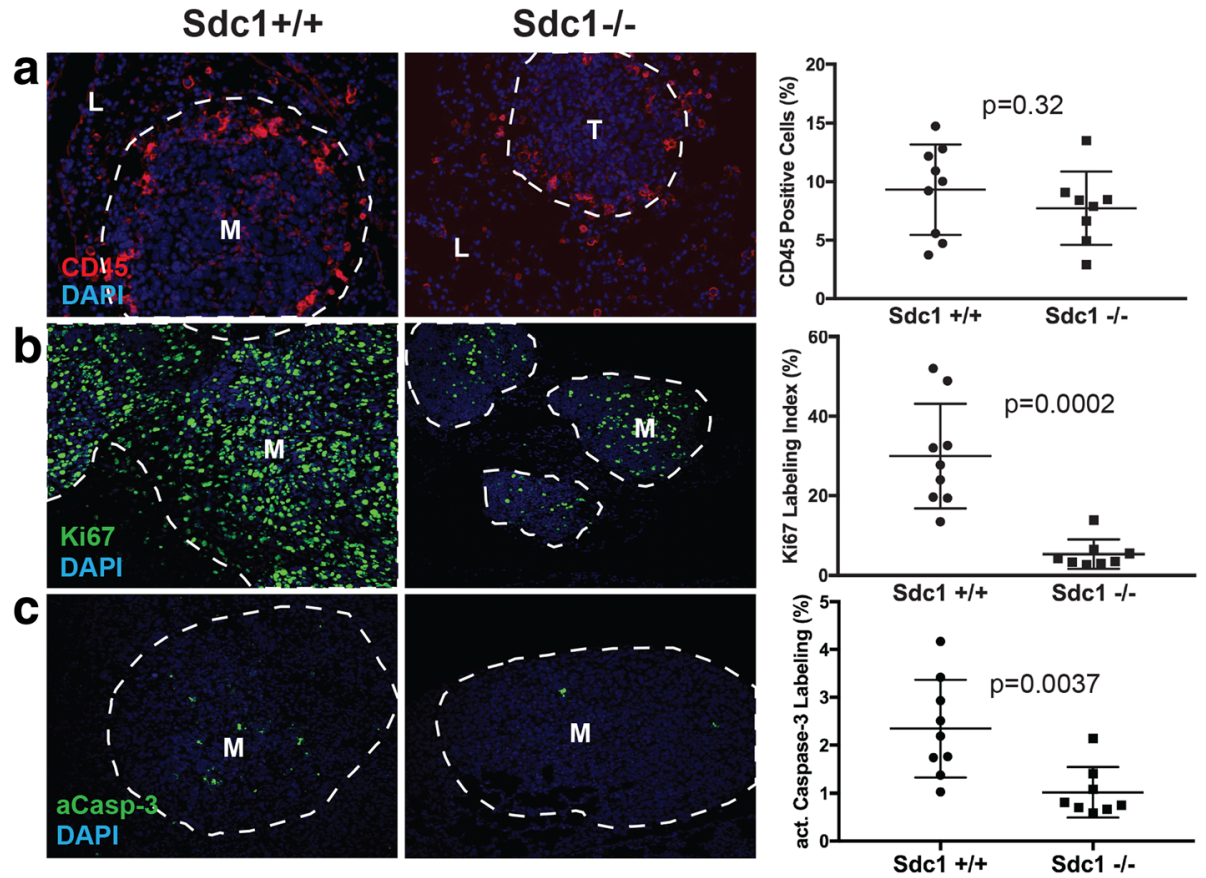

Fig. 4 Characterization of the metastatic microenvironment and metastatic carcinoma cell proliferation/death. Lung metastases arising in Sdc1 $+/+$ and Sdc1-/- mice after tail vein injection of 4T1 cells were analyzed by immunofluorescence or second harmonic generation microscopy and the signal was quantified using ImageJ. a Leukocyte marker CD45 (red) b Proliferation marker Ki67 (green). c Apoptosis marker active caspase 3 (aCasp 3) (green). Original magnification approximately $\times 400$ for all images. Metastatic lesions are outlined with dashed lines. Abbreviations: M, metastasis; L, lung parenchyma; DAPI, 4',6-diamidino-2-phenylindole

The effect of host Sdc1 on metastatic efficiency is abolished in thermo-neutral conditions

Prior work has shown that under typical, mandated animal housing conditions $\left(20-24{ }^{\circ} \mathrm{C}\right)$, Sdc1-deficient mice are susceptible to cold stress, which is linked to a reduced intradermal fat layer and results in the activation of thermogenesis and subsequent development of a $\beta$-adrenergic environment $[38,39]$. These stress responses are relieved when Sdc1-/- animals are transferred to thermo-neutral $\left(30-33{ }^{\circ} \mathrm{C}\right)$ temperatures. This result and the prior observation by Kokolus et al. [17] that ambient temperature influences tumorigenesis, tumor growth and metastasis, persuaded us to repeat the 4T1 fat pad injection experiment in mice subjected to higher ambient temperature; i.e., in thermo-neutral conditions. At a housing temperature of $31{ }^{\circ} \mathrm{C}$, metastatic efficiency was indistinguishable between $\mathrm{Sdc} 1+/+$ and Sdc1-/- animals (Fig. 5). This suggests that the metastasis-promoting or permissive effect of host Sdc1 requires a sub-thermo-neutral environment.

Since Kokolus and coworkers had also reported correlation between housing temperature and intratumoral CD8+ T cells [17], we measured $T$ cell numbers in our fat pad model. Abundant CD4 and CD8 T lymphocytes were identified in 4T1 lung metastases (Additional file 4: Figure S4 A and B). In larger metastases, these

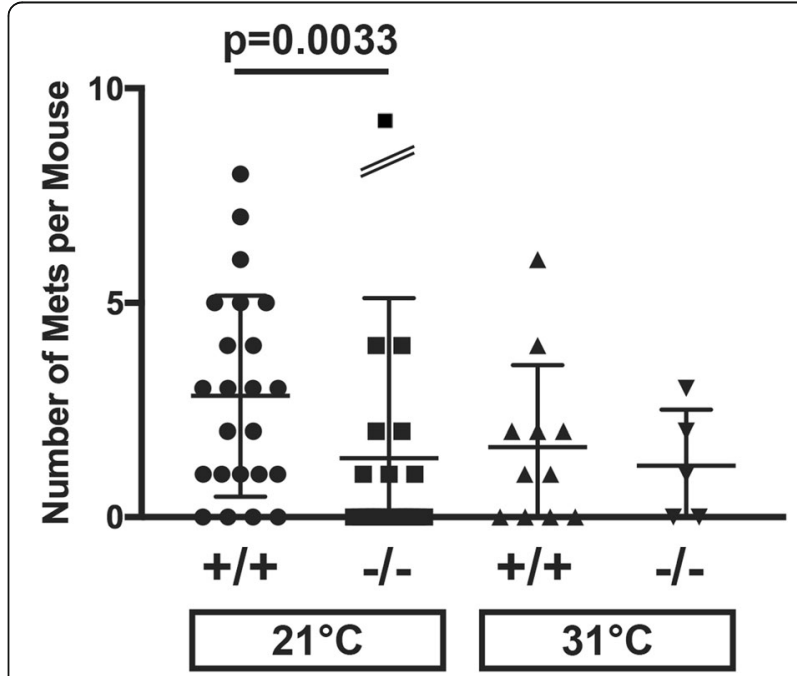

Fig. 5 Effect of ambient temperature on metastatic efficiency. A subset of animals was moved to a housing environment with a higher, thermo-neutral temperature of approximately $31^{\circ} \mathrm{C}$, 2 weeks prior to inoculation and maintained at that temperature throughout the duration of the experiment. The $4 \mathrm{~T} 1$ tumor cells $\left(1 \times 10^{7}\right.$ tumor cells in $10 \mu \mathrm{L}$ serum-free DMEM) were then injected into the fat pads of Sdc1+/+ and Sdc1-/- mice. The animals were sacrificed 30 days after tumor cell inoculation and lung sections were examined for metastases. Shown are numbers of metastases per mouse (one outlier data point of 18 metastases per mouse in Sdc1-/-; $21^{\circ} \mathrm{C}$ group is off scale but is included in mean and SD calculation) 
tumor-infiltrating lymphocytes were located primarily in the tumor periphery (not shown). Consistent with the findings by Kokolus et al., intratumoral CD8+ T cell numbers were increased in mice housed at $31{ }^{\circ} \mathrm{C}$ compared to $21{ }^{\circ} \mathrm{C}$ (Additional file 4: Figure S4 D). In contrast to the Kokolus study, however, we also observed elevated intratumoral $\mathrm{CD} 4+\mathrm{T}$ cells in mice housed at the higher temperature of $31{ }^{\circ} \mathrm{C}$ (Additional file 4: Figure S4 C). Because of the observed effect of housing temperature on intratumoral $\mathrm{T}$ cells and on Sdc1-mediated metastatic efficiency, we examined a possible relationship between Sdc1 deficiency and intratumoral $\mathrm{T}$ cells. The Sdc1 genotype was not significantly associated with intratumoral CD4+ or $\mathrm{CD} 8+\mathrm{T}$ cell numbers at either housing temperature (Additional file 4: Figure S4 E and F). In normal lung tissue, CD4+ and CD8+ cell numbers correlated with neither housing temperature nor Sdc1 status (Additional file 4: Figure S4G, H).

\section{Discussion}

Here we show that host Sdc1 is required for efficient metastasis of mammary carcinoma cells and that the HSPG acts by enhancing the outgrowth of metastatic lesions. The host Sdc1 effect is lost when the animals are placed in thermo-neutral housing conditions.

These observations describe a new pathway by which stromal cell-derived Sdc1 can drive cancer progression by stimulating proliferation of disseminated carcinoma cells at distant organ sites. This is relevant because it ascribes a role to Sdc1 at a critical transition step during the natural history of breast cancer. Local disease is typically controlled with surgical and radiation therapy. However, at the time of diagnosis, carcinoma cells may already have disseminated to distant organ sites, where they may lie dormant for many years. The mechanisms that govern escape from dormancy and outgrowth into clinically apparent metastatic lesions are unknown but are thought to rely on microenvironmental cues from the metastatic niche.

Co-localization studies identified Sdc1 expression in intratumoral fibroblasts within the metastatic niche and failed to detect Sdc1 in endothelial cells or leucocytes, pointing to metastasis-associated fibroblasts as the key cell type, regulating outgrowth. This is consistent with our understanding of stromal Sdc1 activity in primary breast carcinomas. However, airway epithelial cells also express Sdc1 and it is possible that epithelial Sdc1 is responsible for or contributes to metastatic outgrowth in the lung microenvironment. We also cannot rule out the possibility that low levels of Sdc1 expression in cell types other than stromal fibroblasts or airway epithelial cells trigger pathways that stimulate disseminated carcinoma cell proliferation.

The exact mechanism of Sdc1-stimulated metastatic outgrowth is uncertain. Judging from the decreased Ki67 proliferation index seen in Sdc1-/- mice, host Sdc1 stimulates proliferation of disseminated carcinoma cells. In $2 \mathrm{D}$ and in $3 \mathrm{D}$ co-culture models, fibroblast-derived Sdc1 promotes breast carcinoma cell proliferation via paracrine pathways $[6,21]$. In these models, paracrine growth stimulation requires proteolytic cleavage of the Sdc1 core protein resulting in shedding of the Sdc1 ectodomain into the pericellular space. Prior work from several groups including ours revealed matrix metalloproteinase (MMP)14 (aka MT1-MMP) as the obligatory "sheddase" [37, 40]. Other investigators identified heparanase combined with MMP9 as a critical enzyme involved in Sdc1 shedding [41]. In our previously published in vitro model, fibroblast growth factor 2 (FGF2) and stroma-derived factor 1 (SDF-1) complete the paracrine signaling loop that begins with the induction of Sdc1 expression in stromal fibroblasts [21]. Whether or not Sdc1 shedding plays a role in the lung microenvironment in vivo is currently unknown. Sdc1 and its intracellular adapter syntenin are also key molecules in the generation of extracellular vesicles of the exosome class [42]. Furthermore, Sdc1 regulates exosome cargo composition [43]. Since exosomes participate in tumor cell-stroma interactions and exosomes have been shown to prepare the pre-metastatic niche [44], it is conceivable that host Sdc1 stimulates metastasis by modulating exosome production or loading.

Although our results point to an Sdc1 effect on metastatic outgrowth, we cannot rule out the possibility that host Sdc1 levels affect tumor cell extravasation. Götte et al. have shown that Sdc1 deficiency increases the adhesion of leucocytes to retinal endothelium [13]. Any role of endothelial Sdc1 in tumor cell extravasation is speculative at this point.

Sdc1 expression in fibroblasts also leads to the production of ECM with an aligned fiber architecture that is permissive to the directionally persistent migration and invasion of carcinoma cells [23]. ECM fiber alignment in vitro requires activation of the $\alpha v \beta 3$ integrin [45]. Beauvais and coworkers have shown that clustering of Sdc1 on the cell surface results in the assembly of a trimeric complex that also contains an $\alpha v$ containing integrin and insulin-like growth factor 1 receptor (IGF1R) [11]. Ligand-independent activation of the IGF1R triggers inside-out activation of $\alpha v \beta 3$, which could execute ECM fiber alignment. A migration and invasion-permissive microenvironment may enable carcinoma cells to escape microenvironmental niches that suppress growth of disseminated carcinoma cells. Ghajar and colleagues have described a perivascular niche in the lung that traps disseminated mammary carcinoma cells in a dormant state and ascribed a dormancy-inducing activity to endothelial-derived thrombospondin-1 [46].

The dependence of the Sdc1-induced metastasis-promoting effect on sub-thermo-neutral ambient 
temperatures is intriguing. Relief of cold stress does not readily explain the observation since Sdc1-/- animals in sub-thermo-neutral temperature (i.e. typical, mandated housing temperature) are the only ones in this experiment that experience significant cold stress and their metastasis rate does not change when moved to the higher ambient temperature. Kokolus and co-workers report that raising the temperature to thermo-neutral conditions increases CD8+ T-cells number and activity in the primary tumors, while decreasing T-helper cells [17]. In our study, no significant difference was identified in $\mathrm{CD} 4+$ or $\mathrm{CD} 8+$ lymphocytes between $\mathrm{Sdc1}+/+$ and Sdc1-/- animals. Therefore, it is unlikely that $\mathrm{T}$ cells are responsible for mediating the effect of host Sdc1 on metastasis but we cannot entirely rule out differences in lymphocyte activity.

Targeting disseminated breast carcinoma cells during the long period of dormancy or preventing escape from dormancy is a promising therapeutic goal. Sdc1-mediated outgrowth of metastatic lesions may be targetable by interfering with Sdc1 core protein interactions using peptide competitors [47] or by blocking other molecules associated with the Sdc1 pathway such as integrin cell adhesion receptors or receptor tyrosine kinases like IGF1R. However, any therapeutic intervention will require a detailed understanding of the mechanism of Sdc1 action in the metastatic microenvironment.

\section{Conclusions}

In summary, we show that Sdc1 expression is induced in stromal fibroblasts in the lung metastatic microenvironment and that host $\mathrm{Sdc1}$ is required for efficient outgrowth of mammary carcinoma metastases. In thermo-neutral (higher temperature of $31^{\circ} \mathrm{C}$ ) ambient housing conditions, Sdc1 deficiency in the host had no impact on metastasis, suggesting that the Sdc1 effect is temperature-sensitive and likely dependent on mild cold stress. These observations assign an important role to Sdc1 during the late stages of the metastatic cascade, the molecular mechanism of which requires further study.

\section{Additional files}

Additional file 1: Figure S1. Effect of host Sdc1 on metastatic efficiency of E0771 mouse mammary carcinoma cells. E0771 tumor cells $\left(1 \times 10^{7}\right.$ cells in $10 \mathrm{\mu L}$ ) were injected into the exposed 4 th mammary gland as described in "Methods" and mice were killed after 30 days. (A) Metastatic lesion in lung (original magnification $\times 400$; scale bar indicates $100 \mu \mathrm{m}$; M, metastasis; L, lung). (B) Number of metastatic lesions per mouse. Metastases were counted on single histologic sections of both lungs. (C) Metastatic tumor burden expressed as percent lung tissue occupied by metastatic lesions. (D) Average area of metastatic lesions expressed in pixels as measured on histologic sections. (TIF $1153 \mathrm{~kb}$ )

Additional file 2: Figure S2. Characterization of $4 \mathrm{~T} 1$ primary fat pad tumors. The $4 \mathrm{~T} 1$ tumor cells $\left(1 \times 10^{7}\right.$ cells in $\left.10 \mu \mathrm{L}\right)$ were injected into the exposed 4th mammary gland and mice were killed after 30 days. (A) Hematoxylin and eosin (H\&E) stained sections of tumors in fat pad.
Carcinoma cells infiltrate adipose tissue (Ad), which contains benign mammary duct (Duct). Scatter plot graph indicates wet weights of tumors excised from Sdc1+/+ and Sdc1-/- mice. (B) Immunohistochemical $(\mathrm{IHC})$ labeling for proliferation marker Ki67. Graph compares Ki67 labeling index between animal genotypes. (C) $1 \mathrm{HC}$ labeling for endothelial cell marker CD31. Graph compares CD31-positive area between animal genotypes. (D) IHC labeling for alpha smooth muscle actin (aSMA). Graph compares number of aSMA-positive cell clusters between animal genotypes. (E) IHC labeling for macrophage marker F4/80. Graph compares density of F4/80-positive macrophages between animal genotypes. (F) Tumor border imaged by second harmonic generation (SHG) microscopy. White structures indicate fibrillar collagen. Graph compares mean collagen fiber angles relative to tumor boundary between animal genotypes. (TIF 7079 kb)

Additional file 3: Figure S3. Effect of host Sdc1 on later stages of E0771 carcinoma cell metastasis. E0771 tumor cells $\left(1 \times 10^{5}\right.$ tumor cells in $100 \mu \mathrm{L})$ were injected into the tail veins of $\mathrm{C} 57 \mathrm{BL} / 6$ mice, which were killed 15 days later. (A) Metastasis growing around pulmonary vessel (magnification $\times 400$; scale bar indicates $100 \mu \mathrm{m}$; $V$, vessel). (B) Number of metastatic lesions per mouse. Metastases were counted on single histologic sections of both lungs. (C) Metastatic tumor burden expressed as percent lung tissue involved by metastatic lesions. (D) Average area of metastatic lesions expressed in pixels as measured on histologic sections. (TIF $880 \mathrm{~kb}$ )

Additional file 4: Figure S4. Effect of housing temperature and host Sdc1 on T cells within lung metastases. A subset of animals was moved to a housing environment with a thermo-neutral temperature of approximately $31^{\circ} \mathrm{C}, 2$ weeks prior to inoculation and maintained at that temperature throughout the duration of the experiment. The 4T1 mouse mammary carcinoma cells were inoculated into the mammary fat pad as described. Mice were killed after 30 days and sections of lung tissue were labeled with antibodies to CD4 and CD8. CD4+ and CD8+ intratumoral and normal lung lymphocytes were counted as described in "Methods". (A, B) Photomicrographs of adjacent sections of small lung metastasis (M) next to vessel $(V)$ labeled with antibodies to CD4 (A) and CD8 (B) (original magnification $\times 400)$. $(C, D)$ Density of intratumoral lymphocytes in mice segregated by housing temperature expressed as number of cells per megapixel (MP) of metastasis tissue. ( $E, F)$ Density of intratumoral lymphocytes in mice segregated by housing temperature and Sdc1 genotype (same dataset as in $C, D)$. (G, H) Density of lymphocytes in normal lung tissue at distance from any metastases. (TIF 2897 kb)

\section{Abbreviations}

aSMA: Alpha smooth muscle actin; DAPI: 4',6-Diamidino-2-phenylindole; DMEM: Dulbecco's modified Eagle's medium; ER-TR7: Fibroblast antibody; FBS: Fetal bovine serum; H\&E : Hematoxylin and eosin; HS: Heparan sulfate; HSPG: Heparan sulfate proteoglycan; IGF1R: Insulin-like growth factor 1 receptor; MMP: Matrix metalloproteinase; $\mathrm{NIH}$ : National Institutes of Health; RTK: Receptor tyrosine kinase; Sdc1: Syndecan-1; SHG: Second harmonic generation

\section{Acknowledgements}

This article is dedicated to the memory of Patricia Keely, Ph.D., collaborator, mentor and friend. The authors thank the University of Wisconsin Translational Research Initiatives in Pathology Laboratory, in part supported by the UW Department of Pathology and Laboratory Medicine and UWCCC grant P30 CA014520, for use of its facilities and services. We are also grateful to Dr. Alan Rapraeger for providing anti-Sdc1 antibody. The contents do not represent the views of the Dept. of Veterans Affairs or the United States Government.

\section{Funding}

This work was supported by NIH/NCI grant R01 CA107012, DOD grant W81XWH-14-1-0274, and by UWCCC Core Grant P30 CA014520, and also supported in part by award number 101 BX000137 from the Biomedical Laboratory Research and Development Service of the VA Office of Research and Development.

\section{Availability of data and materials}

The datasets used and/or analyzed during the current study are available from the corresponding author on reasonable request. 


\section{Authors' contributions}

Concept and design - AF and CA; acquisition of data - CC, XY, KM, NY, KON and IK; analysis and interpretation - AF, CA, CC, NY, IK and KE; writing - CC and AF. All authors read and approved the final manuscript.

\section{Ethics approval and consent to participate}

This study was performed in strict accordance with the recommendations in the Guide for the Care and Use of Laboratory Animals of the National Institutes of Health. Experimental protocols were approved by the University of Wisconsin School of Medicine and Public Health Animal Care and Use Committee. The number of animals used for each experiment was minimized as much as possible, and every effort was made to reduce the chance of pain or suffering. An exemption category 4 was granted by the Health Science Institutional Review Board of the University of Wisconsin for the study of human biopsy samples. Sections were de-identified prior to staining.

\section{Competing interests}

The authors declare that they have no competing interests.

\section{Publisher's Note}

Springer Nature remains neutral with regard to jurisdictional claims in published maps and institutional affiliations.

\section{Author details}

'Department of Pathology and Laboratory Medicine, University of Wisconsin-Madison, 6051 WIMR, MC-2275, 1111 Highland Avenue, Madison, WI 53705, USA. ${ }^{2}$ Pathology and Laboratory Medicine Service, William S. Middleton Memorial Veterans Hospital, Department of Veterans Affairs Medical Center, Madison, WI, USA. ${ }^{3}$ University of Wisconsin Carbone Cancer Center, Madison, WI, USA. ${ }^{4}$ Laboratory for Optical and Computational Instrumentation, University of Wisconsin-Madison, Madison, WI, USA. ${ }^{5}$ Morgridge Institute for Research, University of Wisconsin-Madison, Madison, WI, USA. 'Department of Oncology, University of Wisconsin-Madison, Madison, WI, USA

\section{Received: 19 December 2017 Accepted: 28 May 2018}

\section{Published online: 05 July 2018}

\section{References}

1. Hüsemann $Y$, Geigl JB, Schubert F, Musiani P, Meyer M, Burghart E, et al. Systemic spread is an early step in breast cancer. Cancer Cell. 2008;13:58-68.

2. Linde N, Fluegen $\mathrm{G}$, Aguirre-Ghiso JA. The relationship between dormant cancer cells and their microenvironment. Adv Cancer Res. 2016;132:45-71.

3. Beauvais DM, Rapraeger AC. Syndecans in tumor cell adhesion and signaling. Reprod Biol Endocrinol. 2004;2:3.

4. Vainio S, Jalkanen M, Bernfield M, Saxén L. Transient expression of syndecan in mesenchymal cell aggregates of the embryonic kidney. Dev Biol. 1992:152:221-32

5. Stanley MJ, Stanley MW, Sanderson RD, Zera R. Syndecan-1 expression is induced in the stroma of infiltrating breast carcinoma. Am J Clin Pathol. 1999;112:377-83.

6. Maeda T, Alexander CM, Friedl A. Induction of syndecan-1 expression in stromal fibroblasts promotes proliferation of human breast cancer cells. Cancer Res. 2004;64:612-21.

7. Jaakkola P, Vihinen T, Määttä A, Jalkanen M. Activation of an enhancer on the syndecan-1 gene is restricted to fibroblast growth factor family members in mesenchymal cells. Mol Cell Biol. 1997:17:3210-9.

8. Määttä A, Jaakkola P, Jalkanen M. Extracellular matrix-dependent activation of syndecan-1 expression in keratinocyte growth factor-treated keratinocytes. J Biol Chem. 1999;274:9891-8

9. Sawaguchi N, Majima T, Iwasaki N, Funakoshi T, Shimode K, Onodera T, et al. Extracellular matrix modulates expression of cell-surface proteoglycan genes in fibroblasts. Connect Tissue Res. 2006;47:141-8.

10. Choi $Y$, Chung $H$, Jung H, Couchman JR, Oh E-S. Syndecans as cell surface receptors: unique structure equates with functional diversity. Matrix Biol. 2011;30:93-9.

11. Beauvais DM, Rapraeger AC. Syndecan-1 couples the insulin-like growth factor-1 receptor to inside-out integrin activation. J Cell Sci. 2010;123:3796-807.
12. Alexander CM, Reichsman F, Hinkes MT, Lincecum J, Becker KA, Cumberledge S, et al. Syndecan-1 is required for Wnt-1-induced mammary tumorigenesis in mice. Nat Genet. 2000;25:329-32

13. Götte M, Joussen AM, Klein C, Andre P, Wagner DD, Hinkes MT, et al. Role of syndecan-1 in leukocyte-endothelial interactions in the ocular vasculature. Invest Ophthalmol Vis Sci. 2002:43:1135-41.

14. Götte M. Syndecans in inflammation. FASEB J. 2003;17:575-91.

15. McDermott SP, Ranheim EA, Leatherberry VS, Khwaja SS, Klos KS, Alexander CM. Juvenile syndecan-1 null mice are protected from carcinogen-induced tumor development. Oncogene. 2007;26:1407-16.

16. Stanford KI, Bishop JR, Foley EM, Gonzales JC, Niesman IR, Witztum JL, et al. Syndecan-1 is the primary heparan sulfate proteoglycan mediating hepatic clearance of triglyceride-rich lipoproteins in mice. J Clin Invest. 2009;119:3236-45.

17. Kokolus KM, Capitano ML, Lee C-T, Eng JW-L, Waight JD, Hylander BL, et al. Baseline tumor growth and immune control in laboratory mice are significantly influenced by subthermoneutral housing temperature. Proc Natl Acad Sci U S A. 2013;110(50):20176-81.

18. Barbareschi M, Maisonneuve P, Aldovini D, Cangi MG, Pecciarini L, Angelo Mauri F, et al. High syndecan-1 expression in breast carcinoma is related to an aggressive phenotype and to poorer prognosis. Cancer. 2003;98:474-83.

19. Anttonen A, Kajanti M, Heikkilä $P$, Jalkanen M, Joensuu H. Syndecan-1 expression has prognostic significance in head and neck carcinoma. Br J Cancer. 1999;79:558-64.

20. Baba F, Swartz K, van Buren R, Eickhoff J, Zhang Y, Wolberg W, et al. Syndecan-1 and syndecan-4 are overexpressed in an estrogen receptor-negative, highly proliferative breast carcinoma subtype. Breast Cancer Res Treat. 2006;98:91-8.

21. Su G, Blaine SA, Qiao D, Friedl A. Shedding of syndecan-1 by stromal fibroblasts stimulates human breast cancer cell proliferation via FGF2 activation. J Biol Chem. 2007;282:14906-15.

22. Maeda T, Desouky J, Friedl A. Syndecan-1 expression by stromal fibroblasts promotes breast carcinoma growth in vivo and stimulates tumor angiogenesis. Oncogene. 2006;25:1408-12.

23. Yang N, Mosher R, Seo S, Beebe D, Friedl A. Syndecan-1 in breast cance stroma fibroblasts regulates extracellular matrix fiber organization and carcinoma cell motility. Am J Pathol. 2011;178:325-35.

24. Miller FR, Medina D, Heppner GH. Preferential growth of mammary tumors in intact mammary fatpads. Cancer Res. 1981;41:3863-7.

25. Schneider CA, Rasband WS, Eliceiri KW. NIH image to ImageJ: 25 years of image analysis. Nat Methods. 2012;9:671-5.

26. Bredfeldt JS, Liu Y, Pehlke CA, Conklin MW, Szulczewski JM, Inman DR, et al. Computational segmentation of collagen fibers from second-harmonic generation images of breast cancer. J Biomed Opt. 2014;19:16007.

27. Davie SA, Maglione JE, Manner CK, Young D, Cardiff RD, MacLeod CL, et al. Effects of FVB/NJ and C57Bl/6J strain backgrounds on mammary tumor phenotype in inducible nitric oxide synthase deficient mice. Transgenic Res. 2007;16:193-201.

28. Ewens A, Mihich E, Ehrke MJ. Distant metastasis from subcutaneously grown E0771 medullary breast adenocarcinoma. Anticancer Res. 2005;25:3905-15

29. De Wever O, Van Bockstal M, Mareel M, Hendrix A, Bracke M. Carcinomaassociated fibroblasts provide operational flexibility in metastasis. Semin Cancer Biol. 2014;25:33-46.

30. Karagiannis GS, Poutahidis T, Erdman SE, Kirsch R, Riddell RH, Diamandis EP. Cancer-associated fibroblasts drive the progression of metastasis through both paracrine and mechanical pressure on cancer tissue. Mol Cancer Res Am Assoc Cancer Res. 2012:10:1403-18.

31. Keikhosravi A, Bredfeldt JS, Sagar AK, Eliceiri KW. Second-harmonic generation imaging of cancer. Methods Cell Biol. 2014;123:531-46.

32. Voyvodic PL, Min D, Liu R, Williams E, Chitalia V, Dunn AK, et al. Loss of syndecan-1 induces a pro-inflammatory phenotype in endothelial cells with a dysregulated response to atheroprotective flow. Journal of biological chemistry. Am Soc Biochem Mol Biol. 2014;289:9547-59.

33. Teng $\mathrm{YH}-\mathrm{F}$, Aquino RS, Park PW. Molecular functions of syndecan-1 in disease. Matrix Biol. 2012;31:3-16.

34. Wculek SK, Malanchi I. Neutrophils support lung colonization of metastasis-initiating breast cancer cells. Nature. 2015;528:413-7.

35. Qian B, Deng Y, Im JH, Muschel RJ, Zou Y, Li J, et al. A distinct macrophage population mediates metastatic breast cancer cell extravasation, establishment and growth. Bereswill S, editor. PloS One. 2009:4:e6562.

36. Williams CB, Yeh ES, Soloff AC. Tumor-associated macrophages: unwitting accomplices in breast cancer malignancy. NPJ Breast Cancer 2016;2:npjbcancer201525. 
37. Su G, Blaine SA, Qiao D, Friedl A. Membrane type 1 matrix metalloproteinasemediated stromal syndecan-1 shedding stimulates breast carcinoma cell proliferation. Cancer Res. 2008;68:9558-65.

38. Tian XY, Ganeshan K, Hong C, Nguyen KD, Qiu Y, Kim J, et al. Thermoneutral housing accelerates metabolic inflammation to potentiate atherosclerosis but not insulin resistance. Cell Metab. 2016;23:165-78.

39. Kasza I, Suh Y, Wollny D, Clark RJ, Roopra A, Colman RJ, et al. Syndecan-1 is required to maintain intradermal fat and prevent cold stress. PLoS Genet. 2014;10:e1004514.

40. Endo K, Takino T, Miyamori H, Kinsen H, Yoshizaki T, Furukawa M, et al. Cleavage of syndecan-1 by membrane type matrix metalloproteinase-1 stimulates cell migration. J Biol Chem. 2003;278:40764-70.

41. Yang Y, MacLeod V, Miao H-Q, Theus A, Zhan F, Shaughnessy JD, et al. Heparanase enhances syndecan-1 shedding: a novel mechanism for stimulation of tumor growth and metastasis. J Biol Chem. 2007:282:13326-33.

42. Baietti MF, Zhang Z, Mortier E, Melchior A, Degeest G, Geeraerts A, et al. Syndecan-syntenin-ALIX regulates the biogenesis of exosomes. Nat Cell Biol. 2012;14:677-85

43. Parimon T, Brauer R, Schlesinger SY, Xie T, Jiang D, Ge L, et al. Syndecan-1 controls lung tumorigenesis by regulating miRNAs packaged in exosomes. Am J Pathol. 2018;188:1094-103.

44. Hoshino A, Costa-Silva B, Shen T-L, Rodrigues G, Hashimoto A, Tesic Mark M, et al. Tumour exosome integrins determine organotropic metastasis. Nature. 2015;527:329-35.

45. Yang N, Friedl A. Syndecan-1-induced ECM fiber alignment requires integrin av 33 and syndecan-1 ectodomain and heparan sulfate chains. Cukierman E, editor. PloS one. 2016;11:e0150132.

46. Ghajar CM, Peinado H, Mori H, Matei IR, Evason KJ, Brazier H, et al. The perivascular niche regulates breast tumour dormancy. Nat Cell Biol. 2013.

47. Rapraeger AC. Synstatin: a selective inhibitor of the syndecan-1-coupled IGF1R-av $\beta 3$ integrin complex in tumorigenesis and angiogenesis. FEBS J. 2013;280:2207-15.

\section{Ready to submit your research? Choose BMC and benefit from:}

- fast, convenient online submission

- thorough peer review by experienced researchers in your field

- rapid publication on acceptance

- support for research data, including large and complex data types

- gold Open Access which fosters wider collaboration and increased citations

- maximum visibility for your research: over $100 \mathrm{M}$ website views per year 